Artículo Original

\title{
Un estudio descriptivo de la presencia, visibilidad y calidad de las revistas de terapia ocupacional
}

\author{
Um estudo descritivo da presença, visibilidade e qualidade dos \\ periódicos de terapia ocupacional
}

\section{A descriptive study of the presence, visibility and quality of occupational therapy journals}

\author{
María Sobrido-Prieto $^{a}$ (D), Miguel-Ángel Talavera-Valverde ${ }^{a}$ (D), Ana-Isabel Souto-Gómez ${ }^{b}$ (D) \\ aUniversidade da Coruña, A Coruña, Galicia, Espanha. \\ bUniversidade de Santiago de Compostela, Santiago de Compostela, Espanha.
}

Cómo citar: Sobrido-Prieto, M., Talavera-Valverde, M. A., \& Souto-Gómez, A. I. (2021). Un estudio descriptivo de la presencia, visibilidad y calidad de las revistas de terapia ocupacional. Cadernos Brasileiros de Terapia Ocupacional, 29, e2918. https://doi.org/10.1590/2526-8910.ctoAO2205

\begin{abstract}
$\underline{\text { Resumen }}$
Con el presente artículo intentamos conocer las revistas actuales de terapia ocupacional, así como la visibilidad y calidad de las mismas. La selección de la muestra se realizó a partir de la base de datos ULRICH y los catálogos Latindex y C17. La búsqueda se completó con un rastreo en la red. Los criterios de inclusión fueron revistas científicas revisadas por pares y activas en el momento de la consulta. Del listado final se analizaron las siguientes variables: visibilidad (se tuvo en cuenta el idioma de publicación y presencia en bases de datos internacionales -ERIC, Psychinfo, Medline, Scopus, OTDBASE, CINAHL and Web of Science), accesibilidad (suscripción, gratuidad y Open Access,) e indicadores de calidad (editorial responsable y factor de impacto). 43 revistas cumplieron los criterios. Respecto a la visiblidad el 69,8\% estaban indexadas en al menos una base de datos, siendo la de mayor representatividad, la base de datos propia de la disciplina, seguidas de las de ciencias de la salud, multidisciplinares y en menor medida las de ciencias sociales. El 25,6\% están incorporadas al movimiento Open Access y el 18,6\% son Free Access. Respecto al prestigio, un 20,9\% incorporadas a JCR y un $41,9 \%$ a SJR. Tras analizar cinco criterios de calidad, únicamente Hong Kong Journal of Occupational Therapy, Irish Journal of Occupational Therapy $y$ Occupational Therapy International los cumplen. Este estudio muestra la realidad de las revistas específicas de la especialidad, así como sus fortalezas y debilidades.
\end{abstract}


Es esperado que conocer esta realidad permitirá mejorar las tasas de producción de conocimiento en terapia ocupacional.

Palabras clave: Terapia Ocupacional, Comunicación Académica, Publicaciones Periódicas como Asunto, Bases de Datos Bibliográficas, Publicaciones Científicas y Técnicas.

\section{$\underline{\text { Resumo }}$}

Com este artigo buscamos conhecer as revistas atuais de terapia ocupacional, bem como sua visibilidade e qualidade. A seleção da amostra foi feita a partir da base de dados ULRICH e dos catálogos Latindex e C17. A pesquisa foi concluída com um rastreamento na web. Os critérios de inclusão foram periódicos científicos revisados por pares e ativos no momento da consulta. Da lista final, foram analisadas as seguintes variáveis: visibilidade (foram considerados o idioma de publicação e a presença em bases de dados internacionais -ERIC, Psychinfo, Medline, Scopus, OTDBASE, CINAHL e Web of Science), acessibilidade (assinatura, acesso gratuito e aberto) e indicadores de qualidade (editora responsável e fator de impacto). Quarenta e três periódicos atenderam aos critérios. Quanto à visibilidade, $69,8 \%$ estiveram indexados em pelo menos uma base de dados, sendo a mais representativa a própria base de dados da disciplina, seguida das ciências multidisciplinares da saúde e, em menor escala, das da Ciências Sociais. 25,6\% estáo incorporados ao movimento de Acesso Aberto e 18,6\% são de Acesso Livre. Em relação ao prestígio, 20,9\% incorporaram-se ao JCR e 41,9\%\% ao SJR. Após analisar cinco critérios de qualidade, só Hong Kong Journal of Occupational Therapy, Irish Journal of Occupational Therapy e a Occupational Therapy International os atendem. Este estudo mostra a realidade dos periódicos específicos da área, bem como seus pontos fortes e fracos. Acredita-se que conhecer essa realidade pode melhorar os índices de produção de conhecimento em terapia ocupacional.

Palavras-chave: Terapia Ocupacional, Comunicação Acadêmica, Publicaçôes Periódicas como Assunto, Bases de Dados Bibliográficas, Publicaçóes Científicas e Técnicas.

\section{Abstract}

With this article, we try to know the current occupational therapy journals, as well as their visibility and quality. The sample selection was made from the ULRICH database and Latindex and C17 catalog. The search was completed with a web search. The inclusion criteria were active and peer-reviewed scientific journals. The variables analyzed were: Visibility (publication language, presence in databases ERIC, Psychinfo, Medline, Scopus, OTDBASE, CINAHL, and Web of Science), accessibility (subscription, free or Open Access), and quality indicators (Journal Citation Reports and Scimago Journal Ranking). 43 journals met the criteria. Visibility $69,8 \%$ were indexed in at least one database, being the most representative, the discipline's database, followed by the health sciences, multidisciplinary and to a lesser extent those of the social sciences. $25,6 \%$ are incorporated into the Open Access movement, and 18,6\% are Free Access. Prestige, $20,9 \%$ are incorporated in JCR and $41,9 \%$ in SJR. After analyzing five quality criteria, only the Hong Kong Journal of Occupational Therapy, Irish Journal of Occupational Therapy, and Occupational Therapy International meet them. This 
study shows the reality of the specific journals of the specialty, as well as their strengths and weaknesses. We hope that knowing this reality we will improve the rates of knowledge production in occupational therapy.

Keywords: Occupational Therapy, Scholarly Communication, Periodicals as Topic, Databases, Bibliographic, Scientific and Technical Publications.

\section{Introducción}

Las revistas científicas son un canal científico importante, a través del cual los avances se comparten, se intercambian opiniones y se proponen teorías. Por ello resulta fundamental conocer las características y funcionamiento de las mismas. Fruto de esta importancia, desde diferentes disciplinas se han llevado a cabo estudios sobre el perfil de estas publicaciones científicas (Sobrido et al., 2017).

Sin embargo, y a pesar de su importancia, en el campo de la terapia ocupacional, son pocos los autores que han realizado un análisis de las mismas. Nos gustaría destacar los estudios de Brown (1997), Brown \& Gutman (2019), Lopes et al. (2016), FernándezMoreno (2012) y Cruz et al. (2019). Si bien existen estudios, estos se encuentran limitados, o bien por ámbito geográfico (Lopes et al., 2016; Fernández-Moreno, 2012) o por los formatos de presentación (Cruz et al., 2019) o revistas de alto impacto.

En definitiva, los estudios mencionados son relevantes para conocer el perfil de las revistas de terapia ocupacional, pero incompletos. Y es que son varios los elementos que quedan por analizar.

En primer lugar, en la actualidad se desconoce la existencia del total de revistas científicas, activas, peer-review y específicas de terapia ocupacional. El último estudio realizado hasta la fecha (Cruz et al., 2019) nos habla de un total de 39 revistas. Varias son las limitaciones que detectamos en este estudio: (a) el concepto de revista. Por una parte, los autores han incluido tanto revistas técnico profesionales como científicas. Por otro lado, limitaron la selección a revistas en línea y (b) el estudio refleja la situación de la publicación en el año 2017. Por ello, hacer un análisis de las revistas actuales y la evolución que han vivido desde su creación resulta fundamental para conocer el papel que la terapia está jugando en la publicación científica.

En segundo lugar, desconocemos la visibilidad y prestigio de estas revistas. Su visibilidad viene marcada por dos elementos: (a) su presencia en las bases de datos, herramientas, fundamentales para recuperar los artículos que responden nuestra duda de un modo rápido, conciso y fiable (Alonso Arroyo \& Aleixandre Benavent, 2012). En el caso de la terapia ocupacional, dado su carácter multidisciplinar (Comité Gestor del Consejo de Colegios de Terapia Ocupacional, 2013), las bases de datos, adquieren mayor importancia; (b) el idioma o idiomas de publicación. Actualmente el inglés es considerado la lengua internacional para la investigación científica, y en algunos casos un requisito fundamental para sobresalir en el ámbito científico (Navarro, 2001). Tal es el impacto que, en determinadas disciplinas, en ocasiones las revistas deciden publicar en inglés, independientemente de su lengua oficial. Así, en el caso de la medicina, son varios los casos de revistas españolas que han decidido publicar los trabajos en inglés. 
Desconocemos si esta situación se da en el caso de la terapia ocupacional, ya que no sabemos los idiomas que se utilizan para dar a conocer los hallazgos.

En cuanto al prestigio, este se ajusta a su calidad y la calidad se puede medir. Aunque existen muchas críticas sobre los indicadores que se usan para cuantificar esta calidad, el factor de impacto es la herramienta más internacionalmente reconocida en cualquier disciplina, y especialmente valorada en el ámbito de la terapia ocupacional (Rodger et al., 2007).

Desde los años 80, existen estudios sobre la producción científica de terapia ocupacional (Brown et al., 2018; Case-Smith \& Powell, 2008; Johnson \& Leising, 1986). La mayoría de ellos se han centrado en un análisis bibliométrico sobre artículos, bien sea de habla inglesa (Case-Smith \& Powell, 2008), o artículos de impacto para la profesión en revistas tanto específicas como no específicas (Brown et al., 2018; Folha et al., 2017). Incluso se han realizado estudios sobre las características de revistas específicas a nivel internacional (Brown, 1997; Brown et al., 2005; Lopes et al., 2016; Oliver, 2009).

Sin lugar a dudas, conocer tanto la producción científica de terapia ocupacional como las características de sus revistas resulta fundamental. Sin embargo, en la actualidad no contamos con estudios que muestren tanto la existencia, visibilidad y/o prestigio de las revistas de la profesión.

Por ello, el objetivo de este estudio es proporcionar información relevante y actualizada sobre las revistas de terapia ocupacional, esto es: (a) conocer la presencia de revistas de terapia ocupacional, (b) su visibilidad y (c) calidad y/o prestigio.

\section{Método}

Para la consecución de nuestro objetivo, llevaremos a cabo un estudio descriptivo de las revistas de terapia ocupacional.

\section{Selección de la muestra}

En agosto de 2020, se llevó a cabo elaboración de un listado de revistas de terapia ocupacional. Para la elaboración de este listado, se tomó como punto de referencia los directorios de publicaciones periódicas más completos y reconocidos, el ULRICH periodicals directory (Exlibris A ProQuest Company, 2000) y tres catálogos, dos internacionales: Latindex (2020), Catálogo de revistas de Revistas Científicas de América Latina, el Caribe España y Portugal (Redalyc, 2020) y otro de ámbito español (Compact Software International, 2020) [C17].

Se tomó como punto de partida el directorio ULRICH periodicals directory (Exlibris. A ProQuest Company, 2000), base de datos de 230.000 publicaciones periódicas de todo el mundo (recoge un total de 200 países). A través de este directorio de revistas se puede consultar la descripción, materias e información. Se seleccionaron las revistas identificadas como "Occupational Health and Safety" en el apartado de materia, por ser la disciplina afín a la terapia ocupacional.

Este listado de revistas se completó con la búsqueda en dos catálogos, el Latindex (2020), Redalyc (Redalyc, 2020) y el C17 (Compact Software International, 2020) 
catálogo colectivo que agrupa las colecciones de publicaciones periódicas de ciencias de la salud de 500 bibliotecas españolas.

Con la intención de no perder ninguna revista, se completó con una búsqueda en google académico. La estrategia de búsqueda fue la siguiente ("occupational therapy" OR Ergohterapy) AND (Journal). Estos términos se buscaron en cuatro idiomas: inglés, portugués, español y francés.

\section{Criterios de selección}

Para la selección de las revistas, y con el fin de comprobar que las revistas seleccionadas eran específicas de la disciplina de terapia ocupacional, los autores establecieron los siguientes criterios: (a) revistas científicas revisadas por pares (peerreview) activas en el momento del estudio; (b) revistas que bien estuviesen elaboradas por instituciones relacionadas con la terapia ocupacional y/o el público al que se dirigía fuesen, entre otros, terapeutas ocupacionales. Este proceso se realizó en septiembre de 2020 por dos autores de modo independiente. Esta información fue obtenida a través de las páginas webs.

\section{Análisis de variables}

Para poder dar respuesta a los dos objetivos planteados en este estudio (la visibilidad y la calidad de las revistas de terapia ocupacional). Esto se llevó a cabo en octubre de 2020, por dos autores de forma independiente, y en el caso de duda, se recurrió al tercer autor.

- Para conocer la evolución de las revistas (años de creación), hemos consultado las bases de datos ULRICH, NLM Catalog y OTDBASE. Además, hemos recurrido a la propia web de la revista en algunos casos;

- Para conocer la calidad y/o prestigio, hemos tenido en cuenta tanto la institución editora responsable, como las principales unidades de medición en el ámbito internacional, Journal Citation Reports (JCR) y Scimago Journal Ranking (SJR), correspondientes al año 2019;

- Para conocer la visibilidad, se tuvieron en cuenta tres elementos: idioma de publicación de la revista, presencia de las mismas en bases de datos: para ello se escogieron tres tipos de bases de datos; especializadas exclusivamente en terapia ocupacional (OTDBASE), especializadas en el ámbito biosanitario (CINAHL, MEDLINE, Psychinfo) y especializadas en el ámbito social y/o multidisciplinar (ERIC, SCOPUS y Web of Science -WOS-), y accesibilidad (Open Access/ Free Access/acceso mediante pago). Para constatar el tipo de accesibilidad hemos revisado la web de las revistas. 


\section{Resultados}

\section{Presencia de revistas de terapia ocupacional}

Se localizaron un total de 98 revistas. De ellas 55 fueron excluidas por no cumplir los criterios. $4,1 \%(\mathrm{n}=4)$ no revisadas por pares y $51(52 \%)$ no estaban vinculadas de modo específico a esta disciplina como son salud ocupacional y laboral con 35(70\%) medicina con cuatro (8\%) y fisioterapia, enfermería y ergonomía con dos revistas (4\%) cada una. El total de revistas que cumplían los criterios establecidos fue de 43 .

\section{Evolución de las revistas}

Con respecto a la evolución de las revistas, podríamos destacar cuatro fases: (a) 1920 1949 con tres revistas (7\%), (b) 1950-1979 con cinco (11,6\%), (c) 1980-1999 con $19(44,2 \%)$ y (d) 2000-2020 con 16 revistas (37,2\%) (Tabla 1$)$.

Tabla 1. Presencia de revistas y año de creación de acuerdo a país de origen $(n=43)$.

\begin{tabular}{|c|c|c|c|}
\hline & Año & Idioma & $\mathbf{P}$ \\
\hline \multicolumn{4}{|l|}{$\overline{\text { AFRICA }}$} \\
\hline \multicolumn{4}{|l|}{ Sudáfrica } \\
\hline South African Journal of Occupational Therapy & 1953 & Inglés & S \\
\hline \multicolumn{4}{|l|}{ Egipto } \\
\hline Occupational Therapy International & 1994 & Inglés & $\mathrm{T}$ \\
\hline \multicolumn{4}{|l|}{ AMERICA } \\
\hline \multicolumn{4}{|l|}{ Canadá } \\
\hline Canadian Journal of Occupational Therapy & 1933 & Ing/Fran & $\mathrm{B}$ \\
\hline Occupational Therapy Now & 1999 & Ing/Fran & $\mathrm{B}$ \\
\hline \multicolumn{4}{|l|}{ Estados Unidos de América } \\
\hline American Journal of Occupational Therapy & 1922 & Inglés & $\mathrm{B}$ \\
\hline Annals of International Occupational Therapy & 2018 & Inglés & $\mathrm{T}$ \\
\hline Journal of Occupational Therapy Education & 2017 & Inglés & $\mathrm{T}$ \\
\hline Occupational Therapy in Mental Health & 1980 & Inglés & $\mathrm{T}$ \\
\hline Occupational Therapy, Schools and Early Intervention & 2008 & Inglés & $\mathrm{T}$ \\
\hline OTJR: Occupation, Participation and Health & 1981 & Inglés & $\mathrm{T}$ \\
\hline The Open journal of occupational therapy & 2012 & Inglés & $\mathrm{T}$ \\
\hline Physical and Occupational Therapy in Geriatrics & 1980 & Inglés & $\mathrm{T}$ \\
\hline Physical and Occupational Therapy in Pediatrics & 1980 & Inglés & $\mathrm{B}$ \\
\hline \multicolumn{4}{|l|}{ Argentina } \\
\hline Revista Argentina de Terapia Ocupacional & 2015 & Castellano & S \\
\hline \multicolumn{4}{|l|}{ Brasil } \\
\hline Cadernos Brasileiros de Terapia Ocupacional & 1990 & $\mathrm{P} / \mathrm{C} / \mathrm{I}$ & $\mathrm{T}$ \\
\hline Revista de Terapia Ocupacional de la USP & 1990 & Portugués & \\
\hline Revista Interinstitucional Brasileira Terapia Ocupacional & 2016 & Portugués & $\mathrm{T}$ \\
\hline
\end{tabular}


Tabla 1. Continuación...

\begin{tabular}{|c|c|c|c|}
\hline & Año & Idioma & $\mathbf{P}$ \\
\hline \multicolumn{4}{|l|}{ Chile } \\
\hline Revista Chilena de Terapia Ocupacional & 2001 & Castellano & A \\
\hline Revista de Estudiantes de Terapia Ocupacional & 2014 & Castellano & $A^{*}$ \\
\hline \multicolumn{4}{|l|}{ Colombia } \\
\hline Revista Ocupación Humana & 1984 & Castellano & S \\
\hline \multicolumn{4}{|l|}{ ASIA } \\
\hline \multicolumn{4}{|l|}{ Israel } \\
\hline Israeli Journal of Occupational Therapy & 1991 & Hebreo & $\mathrm{T}$ \\
\hline \multicolumn{4}{|l|}{ India } \\
\hline Indian Journal of Occupational Therapy & 1995 & Inglés & $\mathrm{C}$ \\
\hline Indian Journal Physiotherapy and Occupational Therapy & 2006 & Inglés & $\mathrm{T}$ \\
\hline International Journal Physiotherapy and Occupational Therapy & 2015 & Inglés & $S$ \\
\hline Physiotherapy and Occupational Therapy Journal & 2008 & Inglés & $\mathrm{B}$ \\
\hline \multicolumn{4}{|l|}{ China } \\
\hline Hong Kong Journal of Occupational Therapy & 1985 & Inglés & S \\
\hline \multicolumn{4}{|l|}{ Corea } \\
\hline Journal of Korean Society of Occupational Therapy & 1993 & Coreano & $\mathrm{T}$ \\
\hline \multicolumn{4}{|l|}{ Japón } \\
\hline Asian Journal of Occupational Therapy & 2001 & Inglés & A \\
\hline Japanese Occupational Therapy Research & 1981 & Japonés & B \\
\hline
\end{tabular}

\section{EUROPA}

\begin{tabular}{|c|c|c|c|}
\hline \multicolumn{4}{|l|}{ Alemania } \\
\hline Ergoscience & 2006 & Alemán & $\mathrm{C}$ \\
\hline Ergotherapie und Rehabilitation & 1962 & Alemán & M \\
\hline \multicolumn{4}{|l|}{ España } \\
\hline Revista Asociación Española de Terapia Ocupacional & 1987 & Castellano & A \\
\hline TOG. Terapia Ocupacional Galicia* & 2004 & Castellano & S \\
\hline \multicolumn{4}{|l|}{ Francia } \\
\hline ErgOThérapies & 2001 & Francés & S \\
\hline Revue Francophone de Recherche en Ergothérapie & 2015 & Francés & S \\
\hline \multicolumn{4}{|l|}{ Holanda } \\
\hline Journal of Occupational Rehabilitation & 1991 & Inglés & $\mathrm{T}$ \\
\hline \multicolumn{4}{|l|}{ Irlanda } \\
\hline Irish Journal of Occupational Therapy & 1992 & Inglés & S \\
\hline \multicolumn{4}{|l|}{ Suecia } \\
\hline Scandinavian Journal of Occupational Therapy & 1994 & Inglés & $8^{*}$ \\
\hline \multicolumn{4}{|l|}{ Reino Unido } \\
\hline British Journal of Occupational Therapy & 1938 & Inglés & M \\
\hline Occupational Therapy in Health Care & 1984 & Inglés & $\mathrm{T}$ \\
\hline World Federation Occupational Therapists Bulletin & 1978 & Inglés & S \\
\hline
\end{tabular}


Tabla 1. Continuación...

\begin{tabular}{|c|c|c|c|}
\hline & Año & Idioma & $\mathbf{P}$ \\
\hline \multicolumn{4}{|l|}{ OCEANÍA } \\
\hline \multicolumn{4}{|l|}{ Australia } \\
\hline Australian Occupational Therapy Journal & 1951 & Inglés & $\mathrm{B}$ \\
\hline \multicolumn{4}{|l|}{ Nueva Zelanda } \\
\hline New Zealand Journal of Occupational Therapy & 1990 & Inglés & $\mathrm{T}$ \\
\hline \multicolumn{4}{|c|}{$\begin{array}{l}\text { Año: año de creación de la revista; P: Periodicidad; P/C/I: Portugués/Castellano/Inglés; Ing/Fran: Inglés/Francés; } \\
\text { Cast/Port: Castellano/Portugués. Revista de Terapia Ocupacional de la USP: Revista de Terapia Ocupacional da } \\
\text { Universidade de São Paulo. A: anual; B: bimestral; C: cuatrimestral; M: mensual; S: semestral; T: trimestral; A*: } \\
\text { anual desde el año 2019; 8*: ocho números al año. TOG. Terapia Ocupacional Galicia*: TOG (A Coruña). }\end{array}$} \\
\hline
\end{tabular}

\section{Distribución geográfica}

América es el continente con mayor representación con un 41,9\% (Norteamérica con 25,6\% y 16,3\% en América Latina), seguido de Europa (27,9\%), Asia (20,9\%), y en menor medida Oceanía y África con un $4,7 \%$ cada uno respectivamente (Tabla 1 ).

En cuanto a los países, 22 aglutinan las 43 revistas de este estudio. Estados Unidos de América se encuentra a la cabeza (20,9\%), seguido de la India (9,3\%) (Tabla 1). De las nueve revistas que aparecieron en esta última década, sigue predominando Estados Unidos de América frente al resto con tres publicaciones.

\section{Visibilidad de las Revistas}

\section{Idioma}

Predominan las publicaciones en inglés $(58,1 \%)$ seguido del castellano (14\%), aunque no existe una significación estadística frente al resto (Tabla 1). Tres revistas (7\%) ofrecen sus contenidos en varios idiomas. Son los casos de Canadian Journal of Occupational Therapy y Occupational Therapy Now por su origen geográfico y Cadernos Brasileiros de Terapia Ocupacional, ofrece hasta tres idiomas (portugués, castellano e inglés).

\section{Presencia en bases de datos}

Las bases de datos son la principal herramienta de consulta de investigadores y profesionales cuando buscan información científica. La presencia de las revistas en estas bases de datos aporta visibilidad a las publicaciones.

Dado el carácter sociosanitario de la terapia, hemos analizado bases de datos que fuesen, de reconocido prestigio, y que abarcasen diferentes áreas científicas; terapia ocupacional (OTDBASE), especializadas en el ámbito biosanitario (CINAHL, MEDLINE, Psychinfo) y especializadas en el ámbito social y/o multidisciplinar (ERIC, SCOPUS y WOS.

El 69,8\% de las revistas tienen presencia en alguna de las bases de datos consultadas. La base de datos con mayor representación es OTDBASE (26), seguida de CINAHL (24), Scopus (18), Medline (17), WOS (15), Psychinfo (9), y ERIC, que cuenta con una revista de la disciplina. 
En lo que respecta al solapamiento en las bases de datos, el 9,3\% de las revistas se encuentran en una sola base de datos, en su mayor parte en OTDBASE. Por el contrario, el 30,2\% no están disponibles en ninguna base de datos. Frente a esta falta de cobertura de la especialidad, el 18,6\% están indexadas en seis bases de datos de modo simultáneo. Ninguna revista se encuentra en las siete bases de datos consultadas (Tabla 2).

Tabla 2. Solapamiento de revistas de terapia ocupacional en las bases de datos revisadas $(n=43)$.

\begin{tabular}{|c|c|c|c|c|c|c|c|}
\hline & $\mathrm{C}$ & $\mathbf{E}$ & $\mathbf{M}$ & $\mathbf{O}$ & $\mathbf{P}$ & $S$ & W \\
\hline \multicolumn{8}{|l|}{ En seis bases de datos } \\
\hline \multicolumn{8}{|l|}{ American Journal of Occupational Therapy } \\
\hline \multicolumn{8}{|l|}{ Australian Occupational Therapy Journal } \\
\hline \multicolumn{8}{|l|}{ British Journal of Occupational Therapy } \\
\hline \multicolumn{8}{|l|}{ Canadian Journal of Occupational Therapy } \\
\hline \multicolumn{8}{|l|}{ Occupational Therapy in Health Care } \\
\hline \multicolumn{8}{|l|}{ Occupational Therapy International } \\
\hline \multicolumn{8}{|l|}{ OTJR: Occupation, Participation \& Health } \\
\hline \multicolumn{8}{|l|}{ Physical and Occupational Therapy in Geriatrics } \\
\hline \multicolumn{8}{|l|}{ En cinco bases de datos } \\
\hline \multicolumn{8}{|l|}{ Journal of Occupational Rehabilitation } \\
\hline \multicolumn{8}{|l|}{ Occupational Therapy in Mental Health } \\
\hline Physical and Occupational Therapy in Pediatrics & & & & & & & \\
\hline Scandinavian Journal of Occupational Therapy & & & & & & & \\
\hline
\end{tabular}

\section{En cuatro bases de datos}

Cadernos Brasileiros de Terapia Ocupacional

Hong Kong Journal of Occupational Therapy

Occupational Therapy, Schools, Early Intervention

\section{En tres bases de datos}

Ergotherapie \& Rehabilitation

Indian Journal Physiotherapy Occupational Therapy

Irish Journal of Occupational Therapy

Occupational Therapy Now

The Open journal of occupational therapy

\section{En dos bases de datos}

Ergoscience

Indian Journal of Occupational Therapy

Revue Francophone de Recherche en Ergothérapie

New Zealand Journal of Occupational Therapy

South African Journal of Occupational Therapy

\section{En una base de datos}

Asian Journal of Occupational Therapy 
Tabla 2. Continuación...

$\begin{array}{lllllll}\text { C } & \text { E } & \text { M } & \text { O } & \text { P } & \text { S } & \text { W }\end{array}$

\begin{tabular}{l}
\hline ErgOThérapies \\
\hline Israeli Journal of Occupational Therapy \\
\hline TOG. Terapia Ocupacional Galicia* \\
\hline World Federation of Occupational Therapists Bulletin \\
\hline En ninguna base de datos \\
\hline Annals of International Occupational Therapy \\
\hline TJPRC:IJPOT \\
\hline Journal of Korean Society of Occupational Therapy \\
\hline Journal of Occupational Therapy Education \\
\hline Physiotherapy and Occupational Therapy Journal \\
\hline Revista Argentina de Terapia Ocupacional \\
\hline Revista Asociación Española de Terapia Ocupacional \\
\hline Revista Chilena de Terapia Ocupacional \\
\hline Revista de Estudiantes de Terapia Ocupacional \\
\hline Revista de Terapia Ocupacional de la USP \\
\hline Revista Interinstitucional Brasileira Terapia Ocupacional \\
\hline Revista Ocupación Humana \\
\hline Japanese Occupational Therapy Research \\
\hline
\end{tabular}

C: CINAHL; E: ERIC; M: Medline; O: OTDBASE; P: Psycinfo; S: Scopus; W: WOS: Web of Science; TOG. Terapia Ocupacional Galicia*: TOG (A Coruña).

\section{Accesibilidad de la revista}

El 55,8\% de las revistas analizadas ofrecen acceso a sus contenidos previa suscripción, frente al 44,2\% que permiten el acceso a sus contenidos.

Revistas Free Access (18,6\%): Asian Journal of Occupational Therapy en 2001, Revista de Terapia Ocupacional de la USP en 2002, Indian Journal Physiotherapy and Occupational Therapy en 2006, South African Journal of Occupational Therapy en 2010, Journal of Korean Society of Occupational Therapy en 2013, Revista de Estudiantes de Terapia Ocupacional en 2014, Revista Argentina de Terapia Ocupacional en 2015 y Annals of International Occupational Therapy en 2018.

Revistas Open Access (25,6\%). Si bien el acceso Open Access, es mayor que el Free Access, este aumento ha sido tardío ya que entre 2000-2009 tan solo un 9,1\% se incorporaban a este movimiento (TOG. Terapia Ocupacional Galicia en 2005) frente al 90,9\% que lo hacía entre 2010-2020 (Cadernos Brasileiros de Terapia Ocupacional en 2013, The Open Journal of Occupational Therapy en 2013, Hong Kong Journal of Occupational Therapy en 2016, Occupational Therapy International en 2017, Revue Francophone de Recherche en Ergothérapie en 2017, Revista Chilena de Terapia Ocupacional en 2018, Revista Interinstitucional Brasileira Terapia Ocupacional en 2018, Revista Ocupación Humana en 2018 y Journal of Occupational Therapy Education en 2019). 
Si bien las revistas Open Access ofrecen acceso libre y gratuito a sus lectores, no siempre su publicación es gratuita. Así, de las revistas Open Access, tres exigen un pago por publicación. Son los casos de Cadernos Brasileiros de Terapia Ocupacional salvo para artículos escritos en español o inglés, Occupational Therapy International y The Open Journal of Occupational Therapy

\section{Calidad y/o Prestigio}

\section{Instituciones editoras responsables}

Las revistas de terapia ocupacional están repartidas en tres categorías: (a) Editoriales comerciales $(53,5 \%)$ : de estas editoriales, las que mayor número de revistas gestionan son Routledge Taylor \& Francis Onlines (30,4\%), y SAGE Journals (17,4\%); (b) Revistas avaladas por sociedades científicas y/o colegios profesionales (27,9\%): el máximo exponente de este colectivo se encuentra en las sociedades Europeas (41,7\%) frente a las americanas (Latinoamérica 19,7\% y Norteamérica 16,7\%), y en menor medida de las Africanas y Oceanía; (c) Revistas avaladas por universidades (18,2\%): destacan los países latinoamericanos (62,5\% de las revistas) (Figura 1).

\begin{tabular}{|c|c|}
\hline \multicolumn{2}{|l|}{ Empresa } \\
\hline Annals of International Occupational Therapy & Healio \\
\hline Asian Journal of Occupational Therapy & J-STAGE \\
\hline Australian Occupational Therapy Journal & Wiley Online Library \\
\hline British Journal of Occupational Therapy & SAGE Journals \\
\hline Canadian Journal of Occupational Therapy & SAGE Journals \\
\hline Ergotherapie und Rehabilitation & Schulz-Kirchner Verlag GmbH \\
\hline Hong Kong Journal of Occupational Therapy & SAGE Journals \\
\hline Indian Journal of Physiotherapy and Occupational Therapy & Institute of Medico-Legal Publications \\
\hline International Journal of Physiotherapy and Occupational Therapy & Chennai Transstellar Journal Publications* \\
\hline Irish Journal of Occupational Therapy & EMERALD Publishing \\
\hline Israeli Journal of Occupational Therapy & JSTOR \\
\hline Journal of Occupational Rehabilitation & SPRINGER \\
\hline Journal of Occupational Science & Routledge Taylor \& Francis Online \\
\hline Journal of Occupational Therapy, Schools, and Early Intervention & Routledge Taylor \& Francis Online \\
\hline Occupational Therapy in Health Care & Routledge Taylor \& Francis Online \\
\hline Occupational Therapy in Mental Health & Routledge Taylor \& Francis Online \\
\hline Occupational Therapy International & Hindawi Limited \\
\hline OTJR: Occupation, Participation and Health & SAGE Journals \\
\hline Physical and Occupational Therapy in Geriatrics & Routledge Taylor \& Francis Online \\
\hline Physical and Occupational Therapy in Pediatrics & Routledge Taylor \& Francis Online \\
\hline Annals of Physiotherapy and Occupational Therapy Journal & RED Flower Publication Pvt. Ltd \\
\hline Scandinavian Journal of Occupational Therapy & Routledge Taylor \& Francis Online \\
\hline Japanese Occupational Therapy Research & J-STAGE \\
\hline World Federation of Occupational Therapists Bulletin & Routledge Taylor \& Francis Online \\
\hline \multicolumn{2}{|l|}{ Sociedad científica } \\
\hline American Journal of Occupational Therapy & American Asociation of Occupational Therapy \\
\hline Ergoscience & Deutschen Verband der Ergotherapeuten \\
\hline ErgOThérapies & Deutscher Verband der Ergotherapeuten \\
\hline Indian Journal of Occupational Therapy & India Occupational Therapists' Association \\
\hline New Zealand Journal of Occupational Therapy & New Zealand Association of Occupational Therapists \\
\hline Occupational Therapy Now & Canadian Association of Occupational Therapists \\
\hline Revista Argentina de Terapia Ocupacional & Asociación Argentina de Terapistas Ocupacionales \\
\hline Revista Asociación Española de Terapia Ocupacional & Asociación Profesional Española de Terapia Ocupacional \\
\hline Revista Ocupación Humana & Colegio Colombiano de Terapia Ocupacional \\
\hline Revue Francophone de Recherche en Ergothérapie & CARAFE** \\
\hline South African Journal of Occupational Therapy & Occupational Therapy Association of South Africa \\
\hline TOG (A Coruña) & Colegio Oficial de Terapeutas Ocupacionales de Galicia \\
\hline \multicolumn{2}{|l|}{ Universidades } \\
\hline Cadernos Brasileiros de Terapia Ocupacional & Universidade Federal de São Carlos. \\
\hline Journal of Korean Society of Occupational Therapy & Yonsei University \\
\hline Journal of Occupational Therapy Education & Eastern Kentucky University \\
\hline Revista Chilena de Terapia Ocupacional & Universidad de Chile \\
\hline Revista de estudiantes de terapia ocupacional & Universidad Bernardo O'Higgins \\
\hline Revista de Terapia Ocupacional da Universidade de São Paulo & Universidade de São Paulo \\
\hline Revista Interinstitucional Brasileira Terapia Ocupacional & Universidades Federais do Brasil \\
\hline The Open journal of occupational therapy & Western Michigan University \\
\hline
\end{tabular}

Figura 1. Presencia de revistas y editor $(\mathrm{n}=44) .{ }^{*}$ Chennai Transstellar Journal Publications and Research Consultancy Private Ltd; ${ }^{* *}$ CARAFE: Communauté pour l'Avancement de la Recherche Appliquée Francophone en Ergothérapie. 


\section{El factor de impacto}

Este índice mide la repercusión de una revista en la literatura científica y por lo tanto es uno de los indicadores más relevantes en el ámbito de la investigación. La presencia de revistas de terapia ocupacional en JCR es de 20,9\%, todas en la categoría de Rehabilitación. Si analizamos estos datos por cuartiles, en Q1 se encuentra la American Journal of Occupational Therapy, siendo el cuartil de mayor predominancia el Q3 $(44,4 \%)$ (Tabla 3).

Tabla 3. Revistas de terapia ocupacional con factor de impacto $(\mathrm{n}=18)$.

\begin{tabular}{|c|c|c|c|c|c|c|}
\hline & \multicolumn{3}{|c|}{ JCR (2019) } & \multicolumn{3}{|c|}{ SJR (2019) } \\
\hline & AI & Cat & Q & Cat & Sub Cat & Q \\
\hline American Journal of Occupational Therapy & $\mathrm{CT}$ & Rhb & 1 & $\mathrm{HP}$ & Occup Ther & 1 \\
\hline Australian Occupational Therapy Journal & $\mathrm{CT}$ & Rhb & 4 & $\mathrm{HP}$ & Occup Ther & 2 \\
\hline British Journal of Occupational Therapy & $\mathrm{CT}$ & Rhb & 3 & HP & Occup Ther & 2 \\
\hline Cadernos Brasileiros de Terapia Ocupacional & -- & -- & -- & $\mathrm{HP}$ & Occup Ther & 3 \\
\hline Canadian Journal of Occupational Therapy & CT & Rhb & 3 & $\mathrm{HP}$ & Occup Ther & 2 \\
\hline Ergotherapie und Rehabilitation & -- & -- & -- & $\mathrm{HP}$ & Occup Ther & 4 \\
\hline Hong Kong Journal of Occupational Therapy & CT & Rhb & 4 & $\mathrm{HP}$ & Occup Ther & 4 \\
\hline Irish Journal of Occupational Therapy & -- & -- & -- & $\mathrm{HP}$ & Occup Ther & 4 \\
\hline Journal of Occupational Rehabilitation & -- & -- & -- & $\mathrm{HP}$ & Occup Ther & 1 \\
\hline Occupational Therapy, Schools, and Early Intervention & -- & -- & -- & SS & Education & 3 \\
\hline Occupational Therapy in Health Care & -- & -- & -- & Med & Medicine & 3 \\
\hline Occupational Therapy in Mental Health & -- & -- & -- & Med & PubHealth & 3 \\
\hline Occupational Therapy International & CT & Rhb & 4 & Med & Medicine & 3 \\
\hline Occupational Therapy Now & -- & -- & -- & Nurs & ASN & 4 \\
\hline OTJR: Occupation, Participation \& Health & CT & Rhb & 3 & HP & Occup Ther & 1 \\
\hline Physical and Occupational Therapy in Geriatrics & -- & -- & -- & $\mathrm{HP}$ & Rhb & 3 \\
\hline Physical and Occupational Therapy in Pediatrics & CT & Rhb & 2 & HP & Occup Ther & 2 \\
\hline Scandinavian Journal of Occupational Therapy & $\mathrm{CT}$ & Rhb & 3 & Med & PubHealth & 2 \\
\hline
\end{tabular}

JCR: Journal Citation Report; SJR: Scimago Journal Ranking; AI: Área de investigacion; Cat: Categoría; Q: Quartil; CT: Ciencia y Tecnología: Ciencias de la Vida y Biomedicina; HP: Health Professions; SS: Social Sciences; Med: Medicine; Nurs: Nursing; ASN: Advanced and Specialized Nursing; Rhb: Rehabilitación; Occup Ther: Occupational therapy; PubHealth: Publics Health, Environmental and Occupational Health.

En el caso del SJR, la presencia de revistas de terapia ocupacional aumenta a 41,2\%. A excepción de seis casos, predominan la ubicadas en la categoría Health Professions $(66,7 \%)$, y Medicine $(22,2 \%)$ En cuanto a las subcategorías, predomina la de Occupational Therapy (61,1\%), Medicine y Publics Health, Environmental and Occupational Health, con 11,1\% respectivamente. En cuanto a los cuartiles, de las 18 publicaciones SJR, el 33,3\% se sitúan en el Q3, el 27,8\% en el Q2, el 22,2\% en el Q4 y el 16,7\% en el Q1 (Tabla 3). 


\section{Estándares de Calidad Global de las Revistas}

De acuerdo a los criterios establecidos en nuestro estudio (presencia de revistas de terapia ocupacional en bases de datos, visibilidad y calidad y/o prestigio de las mismas), podemos encontrar tres grupos (Tabla 4): (a) El 7\% de las revistas cumplen los cuatro requisitos analizados (Hong Kong Journal of Occupational Therapy, Irish Journal of Occupational Therapy y Occupational Therapy International); (b) El grupo de tres criterios cumplidos es el más numeroso con un 32,6\%; (c) El 14\% no cumple ninguno de los criterios definidos.

Tabla 4. Tabla resumen de las variables analizadas.

$\begin{array}{llll}\text { ID } & \text { BD } & \text { OA } & \text { F:I } \\ \text { Ing }\end{array}$

American Journal of Occupational Therapy

Annals of International Occupational Therapy

Asian Journal of Occupational Therapy

Australian Occupational Therapy Journal

British Journal of Occupational Therapy

Cadernos Brasileiros de Terapia Ocupacional*

Canadian Journal of Occupational Therapy**

Ergoscience

Ergotherapie und Rehabilitation

ErgOThérapies

Hong Kong Journal of Occupational Therapy

Indian Journal of Occupational Therapy

Indian Journal of Physiotherapy and Occupational Therapy

International Journal of Physiotherapy \& Occupational Therapy

Irish Journal of Occupational Therapy

Israeli Journal of Occupational Therapy

Japanese Occupational Therapy Research

Journal of Korean Society of Occupational Therapy

Journal of Occupational Rehabilitation

Journal of Occupational Therapy Education

Journal of Occupational Therapy, Schools, and Early Intervention

New Zealand Journal of Occupational Therapy

Occupational Therapy in Health Care

Occupational Therapy in Mental Health

Occupational Therapy International

Occupational Therapy Now**

OTJR: Occupation, Participation \& Health 
Tabla 4. Continuación...

BD OA F:I

Physical and Occupational Therapy in Geriatrics

Physical and Occupational Therapy in Pediatrics

Physiotherapy and Occupational Therapy Journal

Revista Argentina de Terapia Ocupacional

Revista Asociación Espańola de Terapia Ocupacional

Revista Chilena de Terapia Ocupacional

Revista de Estudiantes de Terapia Ocupacional

Revista de Terapia Ocupacional de la USP

Revista Interinstitucional Brasileira Terapia Ocupacional

Revista Ocupación Humana

Revue Francophone de Recherche en Ergothérapie

Scandinavian Journal of Occupational Therapy

South African Journal of Occupational Therapy

The Open journal of occupational therapy

TOG. Terapia Ocupacional Galicia*

World Federation of Occupational Therapists Bulletin

ID: idioma; Ing: inglés; BD: revista incluida en Bases de datos; OA: revista incluida en Open Access; F:I: revista con factor de impacto JCR o SJR; *Revista que se publica en Portugués/Castellano/Inglés; ${ }^{* *}$ Revista que se publica en Inglés/Francés; Revista de Terapia Ocupacional de la USP: Revista de Terapia Ocupacional da Universidade de São Paulo. *Continuación de la versión impresa. TOG. Terapia Ocupacional Galicia*: TOG (A Coruña).

\section{Discusión}

El objetivo de este estudio era proporcionar información relevante y actualizada sobre las revistas de terapia ocupacional, esto es: (a) conocer la presencia de revistas de terapia ocupacional, (b) su visibilidad y (c) calidad y/o prestigio de las mismas (a) conocer la existencia actual, (b) la visibilidad y (c) calidad de las mismas.

\section{Presencia de Revistas de Terapia Ocupacional}

El número de revistas incluidas en este estudio ha sido de 43. Nuestro estudio supone un aumento de revistas frente el estudio de Cruz et al. (2019). Por una parte, desde 2018, la Revista Asturiana de Terapia Ocupacional - Spain) ha dejado de actualizarse, aun así teniendo en cuenta esta situación el número de revistas aumentó en cinco: Revista Asociación Española de Terapia Ocupacional, Journal of Occupational Rehabilitation, Israeli Journal of Occupational Therapy, Journal of Korean Society of Occupational Therapy $y$ Revista de Estudiantes de terapia ocupacional. Entendemos que esta diferencia no se debe a la aparición de nuevas revistas sino a que posiblemente el estudio de Cruz et al. (2019), por una parte tuvo en cuenta consideradas magazines o publicaciones publicitarias, y por otra no tuvo en cuenta revistas que se encontraran en papel, no tuviesen frecuencia regular de publicación o no tuvieran presencia en alguna base de datos. 
Estamos convencidos de que de que el fortalecimiento de la profesión pasa por la difusión del conocimiento generado en revistas de amplia calidad y reconocidas internacionalmente.

En cualquier caso, en nuestro estudio, el número de revistas es bajo comparado con otras disciplinas afines. Entendemos que varias pueden ser las causas: (a) por una parte, somos conscientes que otras disciplinas tienen una trayectoria investigadora mucho más dilatada como es el caso de la medicina, con 4760 revistas (Exlibris A ProQuest Company, 2000); (b) Sobre todo, una población diana marcadamente superior, el caso de la enfermería cuenta con 233 revistas (Exlibris A ProQuest Company, 2000); (c) Existe una mayor dispersión de nuestras publicaciones en otras revistas por motivos de factor de impacto (Folha et al., 2019).

\section{Evolución}

La evolución de las revistas científicas va, inexorablemente unida a dos circunstancias; la propia evolución de la disciplina, y el desarrollo de las revistas científicas. Así podemos destacar cuatro momentos:

Nacimiento (1920-1949): tres revistas (7\%). La creación de las primeras sociedades (estadounidense, canadiense y británica), llevan a la creación de las primeras revistas. Así, la American Journal of Occupational Therapy, surge tan sólo 5 años después del reconocimiento de la terapia como disciplina, en 1917 (Talavera-Valverde, 2007). Una vez reconocida la profesión, surgen las primeras preocupaciones por la investigación como base para el desarrollo de la disciplina. Producto de esta inquietud, en los años 30 las sociedades científicas británica y canadiense lanzan sus propias revistas.

Crecimiento (1950-1979): cinco revistas (11,6\%) (Australian Occupational Therapy Journal, Ergotherapie und Rehabilitation, Indian Journal of Occupational Therapy, South African Journal of Occupational Therapy y World Federation of Occupational Therapists Bulletin). A partir de los años 50, fruto de la revolución tecnológica, las ciencias biomédicas se expanden en el campo de la investigación y tratan abarcar otras disciplinas. Para la terapia ocupacional esto supuso un desafío. Así esta situación dio paso a la creación de nuevas revistas (tanto de la mano de sociedades científicas como editoriales comerciales). Sin duda, la creación de estas revistas supuso toda una declaración de intenciones de la terapia ocupacional en el campo de la investigación.

Expansión (1980-1999): 19 revistas (44,2\%). Dos son las circunstancias que propiciaron una expansión tan relevante. Por un lado, la entrada de la terapia ocupacional a nivel universitario. Por otro lado, la digitalización de las revistas científicas. La incorporación de las revistas a la web facilitó, en muchos casos una reducción de costes en su edición (Abadal, 2020). Como consecuencia se produjo una mayor difusión y visibilidad.

Consolidación (2000-2020): 16 revistas (37,2\%). En esta época la terapia ocupacional se consolida como disciplina académica (aparición de los Grados universitarios, acceso a los estudios de Doctorado o la investigación financiada, entre otros), lo que genera un aumento de la producción científica, y con ello surge una demanda de nuevas revistas. Así, en estas fechas, las revistas europeas aumentan un 25\%, la revista asiática comienza su expansión y se desarrollan las revistas en castellano como son la Revista Ocupación Humana y Cadernos Brasileiros de Terapia Ocupacional. De hecho, específicamente, esta 
revista mejoró en la indexación por varias razones (decisión de publicar los números solo en línea, publicación de textos en inglés o amplia circulación y acceso a los contenidos) (Lopes et al., 2018).

\section{Distribución revistas de acuerdo al entorno geográfico}

La distribución geográfica de revistas ha resultado bastante desigual. Así, de los 193 países, existentes (Organización de las Naciones Unidas, 2021) solo 22 han visto el desarrollo de al menos una revista.

En este sentido de los cinco continentes el que aglutina mayor cantidad de revistas es América (41,9\%), posiblemente por la mayor tradición histórica de Estados Unidos de América, unida al crecimiento de la terapia ocupacional en los países de Sudamérica.

A América le sigue Europa (27,9\%). Tras la segunda guerra mundial (1939-1945) se produce un avance científico y tecnológico sin precedentes, lo que facilita la creación de nuevas revistas científicas, sobre todo en países donde la contienda se desarrolló.

Por otro lado, la evolución de las revistas en Asia (20,9\%), se realizan de la mano de revistas multidisciplinares y van de la mano de publicaciones conjuntas a la fisioterapia (Indian Journal Physiotherapy and Occupational Therapy, International Journal of Physiotherapy and Occupational Therapy y Physiotherapy and Occupational Therapy Journal).

Tras la segunda guerra mundial, en Oceanía y África (4,7\% respectivamente), emergen dos revistas (Australian Occupational Therapy Journal y South African Journal of Occupational Therapy). Consideramos las causas muy dispares; en el caso de Oceanía, ya existía la revista Australian Journal of Occupational therapy, si bien es cierto que los primeros años de esta revista surgen como un Bulletin of the Australian Association of Occupational Therapists, pronto (1963) pasa a convertirse en una revista científica peer review.

Por otra parte, en África la producción científica no existía hasta el momento en ninguna revista dedicada a la especialidad. Sin lugar a dudas, la aparición de South African Journal of Occupational Therapy supuso un impulso sin precedentes para la terapia ocupacional de este continente, ya que por primera vez podrían publicar artículos dentro de su especialidad y dentro de las prácticas profesionales del país.

En definitiva, creemos necesario alentar a la creación de nuevas revistas de la disciplina, pero, además, deberían desarrollarse siempre que las condiciones se den en continentes diferentes a los actuales (como en Oceanía y África), y pudieran comunicar al resto sus logros, mostrar sus aspectos culturales, contextuales, teóricos y de involucración con la disciplina.

\section{Visibilidad de las Revistas de Terapia Ocupacional}

Son varias las formas en las que se puede medir este indicador en una revista; entre ellas hemos considerado, el idioma de publicación, la presencia en bases de datos y el acceso Open Access o Free Access. 


\section{Idioma}

Como cabía esperar, el idioma viene determinado, en la mayor parte de los casos, por la institución/país de la revista. De modo adicional, en algunos casos, algunas revistas han ofrecido un segundo o tercer idioma para dar mayor difusión.

Así, el idioma predominante de revistas es el inglés $(58,1 \%)$. Entendemos que este dato puede ser debido a: en primer lugar, la mayor parte de las revistas son de ámbito geográfico anglófono (un total de 17 revistas, 39,5\%). Pero, es que en el caso de la mayor parte de las revistas asiáticas ( $10 \%$ del total de las revistas) publican exclusivamente en inglés, a pesar de no ser el idioma original. Entendemos que, como estrategia de difusión y expansión (interés por llegar, no sólo a los lectores locales, sino también a lectores potenciales en todo el mundo). Son los casos de Asian Journal of Occupational Therapy, Hong Kong Journal of Occupational Therapy, Indian Journal of Occupational Therapy $y$ Indian Journal of Physiotherapy and Occupational Therapy).

El castellano (14\%) se configura como la segunda lengua más empleada por las revistas. A excepción de una revista (Cadernos Brasileiros de Terapia Ocupacional) todas ellas proceden de ámbitos geográficos castellano parlante.

Nos gustaría destacar tres revistas que publican en varios idiomas de modo simultáneo. La Canadian Journal of Occupational Therapy y la Occupational Therapy Now tiene duplicidad idiomática por su carácter bilingüe. Quitando estas dos excepciones, sólo la revista Cadernos Brasileiros de Terapia Ocupacional ofrece sus contenidos en tres idiomas, precisamente para dar mayor visibilidad, y difusión a sus contenidos. Si bien somos conscientes del esfuerzo (económico y en recursos humanos) (Lopes et al., 2018) animamos a las revistas a ofrecer sus contenidos en otros idiomas.

\section{Representación en base de datos}

La representación en bases de datos es muy alta $(69,8 \%)$ y positiva para la terapia ocupacional. Los nuevos progresos de la disciplina, pueden verse limitados si las revistas no están incorporadas en las bases de datos internacionales, principal herramienta para estar al día de los avances de la disciplina (Cruz et al., 2019). Si bien los datos de revistas no presentes son bajos $(30,2 \%)$, consideramos necesario un estudio en donde se analicen las causas de exclusión de estas revistas.

Los resultados de nuestro estudio, apuntan a una posible relación entre editoriales comerciales y presencia en bases de datos (el 60\% de las revistas gestionadas por editoriales comerciales, se encuentran presentes en alguna base datos). Esto nos hace pensar que la profesionalización de las prácticas editoriales, facilita obtener los criterios suficientes para acceder a determinadas bases de datos.

En el caso de las nueve revistas en castellano, tan solo dos (22,2\%) se encuentran indexadas en alguna base de datos (Cadernos Brasileiros de Terapia Ocupacional, en cuatro bases de datos, y TOG. Terapia Ocupacional Galicia en una). ¿Por qué una presencia tan baja? En nuestra opinión la mayoría de estas revistas han surgido evidentemente como una necesidad de la disciplina, pero sobre todo a la voluntad de profesionales terapeutas, que, conscientes de la falta de revistas de su campo han elaborado de modo altruista y con mucho esfuerzo estas publicaciones. Esta situación les ha llevado a que en ocasiones no conozcan/no dispongan de recursos para implantar 
criterios que guíen las buenas prácticas en la publicación científica. Además, si tenemos en cuenta los datos del estudio, ninguna revista en castellano está en manos de ninguna empresa editorial, con todo lo que eso conlleva a la hora de no profesionalizar la gestión de la misma. Pueden ser varios los factores que influyen en esta situación. Por un lado el costo de ese proceso que ocasiones es impensable para las organizaciones que gestionan las publicaciones. Por otro y tal y como describe Vasen \& Vilchis (2017), con los sistemas tan estrictos de clasificación para acceso a determinadas bases de datos, sucede que algunas revistas acaben debilitándose por la ausencia de publicaciones. Teniendo en cuenta que el $75 \%$ de las revistas indexadas en Ulrich's son de habla inglesa, las revistas en castellano, están sufriendo de una menor participación frente a las inclinaciones de los autores por publicar en revistas con altos factores de impacto, indexadas en determinadas bases de datos y en otro idioma diferente al castellano.

De las bases de datos analizadas, como era de suponer, la más potente es OTDBASE, especializada precisamente en este ámbito. En segundo lugar, bases de datos especializadas en ciencias de la salud (CINAHL y Medline). En tercer lugar, las bases de datos multidiciplinares (Scopus y WOS). En último lugar las bases de datos de ciencias sociales (ERIC y Psychinfo). Así, de acuerdo a los datos obtenidos la terapia ocupacional tiene una mayor visibilización dentro del ámbito sanitario. Esta situación nos llama la atención, máxime si tenemos en cuenta la diversidad de la disciplina, ya que la terapia ocupacional se entiende como una profesión con un enfoque socio-sanitario, en la medida en que deben basar su conocimiento profesional no solo en condiciones médicas, si no también en aspectos comunitarios, sociales, psicológicos, sociológicos y de las ciencias humanas, entre otros (World Federation of Occupational Therapists, 2016).

\section{Accesibilidad}

El 44,2\% de las revistas ofrecen acceso a los contenidos. Somos conscientes de las dificultades económicas que supone esta opción. El costo asociado a las revistas corre a cargo de la organización patrocinadora, la editorial o, de los autores que envían los artículos.

Queremos destacar la presencia de la terapia ocupacional en el acceso abierto $(25,6 \%)$. Si comparamos estos datos con otras disciplinas, en el caso de la medicina, sólo el $0,28 \%$ de las revistas están adheridas al movimiento Open Access, y en el caso de la enfermería el 23\% (Exlibris A ProQuest Company, 2000). Aunque entendemos que estos datos son orientativos, nuestro estudio avala que la adherencia de la terapia al Open Access es muy alta y positiva ya que puede facilitar la visibilidad de la disciplina. Este avance de la disciplina sin duda, tendrá entre otros, beneficios para: (a) Investigadores. El acceso abierto ofrece la opción de que al tener mayor difusión será mucho más leído y más veces citado; (b) Disciplina. Se agiliza la transferencia del conocimiento, disminuye las dificultades para acceder al conocimiento y facilita la difusión global; (c) Instituciones que acogen profesionales de terapia ocupacional incrementando la visibilidad de estas instituciones y reflejando la actividad investigadora de la universidad facilitando su evaluación.

Dentro de esta opción queremos destacar la presencia de tres revistas Open Access "author pays" (Cadernos Brasileiros de Terapia Ocupacional, Occupational Therapy 
International, The Open Journal of Occupational Therapy) ${ }^{1}$. Esta opción ha sido muy polémica (Abadal, 2017). Y es que de acuerdo a este modelo los costes de revisión y edición de los manuscritos no recaen sobre los lectores o sino sobre los autores o las instituciones que financien su investigación. Sin embargo, no debemos olvidar que en muchos casos, esta es la única opción que permite consolidar las escasas vías de financiación con las que cuentan algunas revistas.

Aunque no hemos podido demostrar una posible relación entre la accesibilidad con un aumento del factor de impacto, animamos a las editoriales a que hagan el esfuerzo de vincular sus revistas a la ciencia abierta, en cualquiera de los modelos existentes. A pesar de la presión que existe por el FI, no podemos olvidar que la accesibilidad no sólo beneficiará a las propias revistas (mayor visibilidad), si no y sobre todo a la profesión.

\section{Calidad y/o Prestigio de las Revistas de Terapia Ocupacional}

Con respecto a la calidad y/o prestigio de la terapia hemos tenido en cuenta los siguientes elementos:

\section{Institución editorial}

Lo adecuado sería que las revistas estuviesen elaboradas y dirigidas por instituciones relacionadas con la disciplina (universidades o sociedades científicas, entre otras). Pero crear y mantener una revista científica supone un gran esfuerzo; que va desde la selección y generación de contenido constante, evaluación de artículos, maquetación y difusión, entre otros aspectos (López-Cózar et al., 2006). Como es de suponer, todas estas tareas requieren un importante equipo humano, a tiempo completo, y una fuerte financiación, de la que no siempre gozan.

Hemos localizado tres tipos de editoriales. En primer lugar, las editoras comerciales $(53,5 \%)$. Al igual que en otras disciplinas, entendemos que esto se debe a los costes generados por las revistas científicas (Larivière et al., 2015). Los costos fijos de las publicaciones científicas disminuyen con la escala de la producción, lo que hace que las editoriales comerciales tengan un mayor margen de beneficios.

La fuerte presencia de editoriales comerciales, tiene sus inconvenientes y ventajas. Como principales inconvenientes, refuerza el control sobre la comunidad científica. Así de las cinco editoriales comerciales que controlan la producción mundial (Reed-Elsevier, Wiley-Black-well, Springer and Taylor and Francis and Sage Publications) (Larivière et al., 2015), dos, Routledge Taylor \& Francis Onlines (30,4\%) y SAGE Journals $(17,4 \%)$, controlan el $50 \%$ de la producción de terapia ocupacional.

Como principal ventaja, creemos que las empresas editoriales aportan profesionalización de roles de gestión editorial. Esto implica por una parte mayor rentabilización de los engranajes de la edición científica; personal especializado y a tiempo completo. La descarga de estas tareas (maquetación, diseño o desarrollo web, entre otras), permite que los editores (generalmente terapeutas ocupacionales), se puedan centrar en la selección y revisión de contenidos. Esto supone una mejora de calidad y una mayor agilidad de manuscritos. El buen hacer de sociedades científicas por

\footnotetext{
${ }^{1}$ Cadernos Brasileiros de Terapia Ocupacional: $\mathrm{R} \$ 1.050$ salvo para artículos escritos en español o inglés; Occupational Therapy International: \$1350; The Open journal of occupational therapy (el autor principal debe pagar una tarifa de procesamiento de $\$ 300$ dólares estadounidenses y cada autor adicional debe pagar $\$ 100$ dólares estadounidenses).
} 
gestionar sus revistas sin contar con una edición profesional, genera que aspectos de este tipo resten proyección y visibilidad a determinados contenidos.

Es por eso que en la medida en que las editoriales comerciales son económicamente más potentes, las posibilidades de cumplir determinados criterios de calidad son mayores y por lo tanto tienen mayor visibilidad (presencia en bases de datos) y prestigio (factor de impacto).

De hecho, dos son los indicadores a tener en cuenta: (a) Si tomamos como aspecto representativo la aparición de la revista en JCR o SJR, todas las publicaciones, salvo tres (American Journal of Occupational Therapy, Cadernos Brasileiros de Terapia Ocupacional y Occupational Therapy y Occupational Therapy Now), son editoriales comerciales; (b) Las tres únicas revistas que cumplen con el mayor número de criterios de las revistas en nuestro estudio (Hong Kong Journal of Occupational Therapy, Irish Journal of Occupational Therapy y Occupational Therapy International), se encuentran gestionadas por editoriales comerciales.

En segundo lugar, las revistas auspiciadas por sociedades científicas (27,9\%). El patrocinio de sociedades es especialmente valorado por cuanto son las instituciones en las que se agrupan investigadores y profesionales de la disciplina. No sólo dan lustro a las revistas si no que son las responsables de la línea editorial. Es por lo que prácticamente todos los modelos de evaluación de publicaciones periódicas científicas tienen muy en cuenta este indicador. Frente a este reconocimiento, no debemos olvidar que la edición de una revista es una tarea que las sociedades deberían asumir, y que no siempre pueden.

Por ese motivo entendemos dos como los principales inconvenientes: (a) la dificultad económica y (b) la dificultad de gestión editorial, que un profesional de la terapia ocupacional no tiene por qué conocer.

De hecho, si bien desconocemos el número de sociedades científicas de terapia ocupacional en todo el mundo, de 194 países, solo 11 ha auspiciado una revista científica. Nos parece una cifra muy baja, máxime si tenemos en cuenta que una sociedad agrupa al colectivo y el exponente de la disciplina en un país o una región. Estos datos implican una baja iniciativa en la investigación y en la difusión de contenidos científicos. Entendemos que una sociedad no apueste por mantener una revista, dados los recursos necesarios, tanto profesionales como económicos.

Creemos que esta puede ser la razón por la que las sociedades han preferido externalizar sus revistas en empresas. Creemos que la profesionalización de la edición ha facilitado el crecimiento de algunas revistas, su inmediatez en tiempos de respuesta y su expansión. Por todo ello consideramos que la situación ideal de las revistas sería la de una fusión entre sociedades científicas (responsables de contenidos) y empresas editoriales (apoyo logístico). Aun así, ejemplos como la American Journal of Occupational Therapy, son meritorios.

Por último, las revistas editadas por universidades son las de menor calado (18,6\%). teniendo en cuenta que las Universidades son centros de generación del conocimiento. Este bajo porcentaje puede venir dado por las dificultades de gestionar una revista, sometidos a la lógica administrativa de las instituciones a las que pertenecen. La gestión de recursos humanos (reemplazo constante de editores), ausencia de conocimiento del proceso editorial, cierto temor por el arbitraje por pares, falta de equipos que ayuden a mejorar la visibilidad, posicionamiento e indexación de la revista.

Nos gustaria destacar el caso de la revista Cadernos Brasileiros de Terapia Ocupacional. La Universidade Federal de São Carlos, ha sido capaz de superar la falta generalizada de inversiones para el desarrollo de publicaciones periódicas (Kellner, 2017), hasta 
posicionarse en determinadas bases de datos e índices de impacto, logrando ser la revista con mayor proyección de ámbito latinoamericano.

\section{Herramientas de calidad}

El 41,3\% de las publicaciones están presentes en alguno de los indicadores de calidad (20,5\% en JCR y 43,2\% en SJR). Las diferencias detectadas entre nuestro estudio, y los de Brown \& Gutman (2019) vienen marcada por la muestra. Y es que Brown \& Gutman (2019) se centran en revistas presentes en el JCR, mientras que en nuestro estudio hemos ampliado el universo a revistas a peer review activas.

En cualquier caso, existe una gran disparidad de resultados JCR frente a SJR. Entendemos que esto no es tanto por la baja calidad de las revistas, si no por los sistemas de medición. Así, en el caso de JCR, la terapia ocupacional no tiene categoría propia y las revistas deben competir con otras incluidas en la categoría de Rehabilitación, lo que resta visibilidad académica y profesional. Esta evidencia ya fue realizada por Lopes et al. (2016). Producto de esta situación, sólo nueve revistas cuentan con factor de impacto y sólo una está situada en el primer cuartil. En el caso del SJR, si existe una categoría específica. Esto se refleja en el número de revistas de terapia presentes (18), donde tres de estas revistas están situadas en el primer cuartil.

El reconocimiento de la terapia ocupacional como categoría propia daría mayor visibilidad a la disciplina y a las revistas incluidas en el JCR. Este hecho queda corroborado con los datos ofrecidos en nuestro estudio.

Tradicionalmente las revistas más valoradas por los terapeutas (Rodger et al., 2007) han sido: American Journal of Occupational Therapy, Australian Occupational Therapy Journal, British Journal of Occupational Therapy, Canadian Journal of Occupational Therapy, OTJR: Occupation, Participation and Health, y Scandinavian Journal of Occupational Therapy. De acuerdo a nuestro estudio, las revistas con mayores estándares de calidad establecidos son tres (Hong Kong Journal of Occupational Therapy, Irish Journal of Occupational Therapy y Occupational Therapy International). Entendemos que, entre otras, dos pueden ser las causas de estos cambios: (a) El estudio de Rodger et al. (2007) data de 2007 y por lo tanto la percepción de los terapeutas haya cambiado a lo largo de estos ańos; (b) La distancia producida entre el ámbito investigador, y el ámbito profesional sobre la calidad de las revistas científicas.

\section{Limitaciones en la Investigación}

Este estudio se ha limitado a las revistas existentes en el momento actual. En ningún caso se ha tratado de realizar un estudio sobre la evolución histórica de las revistas de esta disciplina.

Los cambios de los nombres de revistas a lo largo de su vida, ha sido un hándicap para localizar su año de creación lo que ha llevado a consultar varias fuentes incluidas la de la propia revista para llegar a concretar ese dato.

Las revistas seleccionadas para este estudio se han limitado a aquellas revistas que se englobasen dentro de la materia de terapia ocupacional. Sin embargo, dado el carácter multidisciplinar de la terapia, entendemos que pueden existir muchas revistas de interés para la terapia, que no han sido contempladas en este estudio, como puede ser la Journal of Occupational Science, entre otras. 
Que existan revistas (en activo, peer-review y de la disciplina) que no se encuentren en ninguna base de datos genera dificultades a la hora de localizarlas.

\section{Futuras Líneas de Investigación}

Conocer las estrategias más adecuadas para la visibilidad y prestigio de sus revistas, es un requisito necesario en cualquier profesión. Por esta razón, dos son las líneas de investigación futuras propuestas:

Presencia de revistas en bases de datos. Es fundamental conocer cuáles son los criterios establecidos por las bases de datos analizadas, y determinar cuáles son los criterios que las revistas cumplen y/o incumplen. Solo de este modo podremos conseguir una mayor visibilidad y prestigio de la terapia ocupacional.

Open Access. Sabemos que el efecto de este movimiento aporta visibilidad y aumento de prestigio (mayor número de citas o agilización de la transferencia del conocimiento, entre otros), incluso esto se ha demostrado en varias disciplinas, pero no conocemos ningún estudio aplicado a la terapia ocupacional. Por ese motivo con esa posible investigación podríamos ver cuál es el potencial beneficio que tiene este movimiento sobre la disciplina.

\section{Conclusiones}

El número de revistas de terapia ocupacional es muy bajo, si lo comparamos con otras disciplinas afines. Esto hace que la posibilidad de publicar en textos disciplinares sea menor.

La época que transcurre desde los comienzos de los años 80 hasta finales de los 90 , ha sido prolífica en la creación de revistas. En cambio desde el 2000 hasta la actualidad, esta evolución ha ido decreciendo. Para el avance de la profesión y de la investigación de la terapia ocupacional es importante que se generen nuevas revistas científicas que aumenten la posibilidad de publicar nuevas investigaciones, y sobre todo que representen nuevos contextos culturales y geográficos.

De acuerdo a su presencia en bases de datos, la terapia ocupacional tiene una mayor visibilidad hacia el ámbito sanitario. Consideramos importante realizar estudios que ahonden en este sentido, esto es, conocer las líneas temáticas más habituales de la disciplina, y con ello los perfiles preponderantes en la profesión.

La visibilidad (idioma, presencia en bases de datos y accesibilidad) cumple niveles aceptables. Creemos necesario que las publicaciones sean capaces de ir desarrollando sus cuerpos editoriales, ajustándose cada vez más a los mínimos establecidos por las diferentes bases de datos y adhiriéndose progresivamente al movimiento Open Access.

La aportación de las editoriales comerciales a las revistas científicas facilita la profesionalización de estas. Aumentan su visibilidad y calidad, posibilitando el crecimiento y la obtención de ciertos criterios de calidad con mayor rapidez, salvo algunas excepciones. De todas formas y ante los beneficios e inconvenientes de las múltiples opciones que hay, apostamos por una fusión entre sociedades científicas y editoriales comerciales, en las que se pueda tener cabida editores profesionales y terapeutas ocupacionales. 


\section{Referencias}

Abadal, E. (2017). Las revistas científicas en el contexto del acceso abierto. In E. Abadal (Ed.), Revistas cientificas: situación actual y retos de futuro (pp. 181-195). Barcelona: Universitat de Barcelona.

Abadal, E. (2020). Un libro para explicar los marcos y los retos de las revistas científicas. In L. Silveira \& F. Couto (Eds.), Gestão editorial de periódicos científicos: tendências e boas práticas (pp. 11-17). Florianópolis: BU Publicaçóes/UFSC.

Alonso Arroyo, A., \& Aleixandre Benavent, R. (2012). Importancia para una revista científica de encontrarse incluida en las grandes bases de datos internacionales. Revista de Patología Respiratoria, 15(4), 101-103. http://dx.doi.org/10.1016/S1576-9895(12)70153-4.

Brown, G. T. (1997). Characteristics of occupational therapy journals of WFOT member countries: a descriptive survey. Occupational Therapy International, 4(2), 116-134. http://dx.doi.org/10.1002/oti.51.

Brown, G. T., Rodger, S., \& Brown, A. (2005). Publication practices of English language occupational therapy journals. British Journal of Occupational Therapy, 68(2), 85-92. http://dx.doi.org/10.1177/030802260506800205.

Brown, T., \& Gutman, S. A. (2019). Impact factor, eigen factor, article influence, scopus SNIP, and SCImage journal rank of occupational therapy journals. Scandinavian Journal of Occupational Therapy, 26(7), 475-483. http://dx.doi.org/10.1080/11038128.2018.1473489.

Brown, T., Gutman, S. A., Ho, Y. S., \& Fong, K. N. K. (2018). A bibliometric analysis of occupational therapy publications. Scandinavian Journal of Occupational Therapy, 25(1), 1-14. http://dx.doi.org/10.1080/11038128.2017.1329344.

Case-Smith, J., \& Powell, C. A. (2008). Research literature in occupational therapy, 2001-2005. The American Journal of Occupational Therapy, 62(4), 480-486. http://dx.doi.org/10.5014/ajot.62.4.480.

Comité Gestor del Consejo de Colegios de Terapia Ocupacional. Asociaciones Profesionales de Terapia Ocupacional. (2013). Informe técnico sobre terapia ocupacional. Madrid: Comité Gestor del Consejo de Colegios de Terapia Ocupacional y Asociaciones Profesionales de Terapia Ocupacional.

Compact Software International. (2020). Catálogo C17. Recuperado el 10 de enero de 2020, de https://www.c17.net

Cruz, D. M. C., Costa, J. D., Veiga, J., Manzini, M. G., \& Folha, O. A. A. C. (2019). Revistas de terapia ocupacional disponibles en línea: estudio descriptivo. Revista de la Facultad de Medicina, 67(3), 437442. http://dx.doi.org/10.15446/revfacmed.v67n3.66778.

Exlibris A ProQuest Company. (2000). Ulrich's periodicals directory. Recuperado el 10 de enero de 2020, de https://www.library.ucsb.edu/research/db/338

Fernández-Moreno, A. (2012). Publicaciones seriadas de la terapia ocupacional en Latinoamérica. Cadernos Brasileiros de Terapia Ocupacional, 20(2), 287-292. http://dx.doi.org/10.4322/cto.2012.030.

Folha, O. A. A. C., Folha, D. R. S. C., Cruz, D. M. C., Barba, P. C. S. D., \& Emmel, M. L. G. (2019). Caracterização de publicaçóes científicas sobre terapia ocupacional em periódicos não específicos da profissão no período de 2004 a 2015. Cadernos Brasileiros de Terapia Ocupacional, 27(3), 650-662. http://dx.doi.org/10.4322/2526-8910.ctoAO1700.

Folha, O. A. C., Cruz, D. M. C., \& Emmel, M. L. G. (2017). Mapeamento de artigos publicados por terapeutas ocupacionais brasileiros em periódicos indexados em bases de dados. Revista de Terapia Ocupacional da Universidade de São Paulo, 28(3), 358-367. http://dx.doi.org/10.11606/issn.22386149.v28i3p358-367.

Johnson, K. S., \& Leising, D. J. (1986). The literature of occupational therapy: a citation analysis study. The American Journal of Occupational Therapy, 40(6), 390-396. http://dx.doi.org/10.5014/ajot.40.6.390.

Kellner, A. W. A. (2017). Editors of Brazilian journals: a hard life that is getting harder! Anais da Academia Brasileira de Ciências, 89(1), 1-2. http://dx.doi.org/10.1590/0001-37652017891.

Larivière, V., Hustein, S., \& Mongeon, P. (2015). The oligopoly of academic publishers in the digital era. PLoS One, 10(6), e0127502. http://dx.doi.org/10.1371/journal.pone.0127502.

Latindex. (2020). Latindex. Sistema Regional de Información en Linea para Revistas Cientificas de América Latina, el Caribe, España y Portugal. Recuperado el 15 de noviembre de 2020, de https://www.latindex.org/latindex/inicio. 
Lopes, R. E., Cruz, D. M. C., \& Malfitano, A. P. S. (2018). Em tempos difíceis, compartilhando boas notícias em meio a muito trabalho. Cadernos Brasileiros de Terapia Ocupacional, 26(3), 509-510. http://dx.doi.org/10.4322/2526-8910.ctoED2632.

Lopes, R. E., Duarte, M. L. M. C., Pereira, B. P., Oliver, F. C., \& Malfitano, A. P. S. (2016). A divulgação do conhecimento em terapia ocupacional no Brasil: um retrato dos seus periódicos. Cadernos de Terapia Ocupacional da UFSCar, 24(4), 777-789. http://dx.doi.org/10.4322/0104-4931.ctoAO0798.

López-Cózar, E. D., Ruiz-Pérez, R., \& Jiménez-Contreras, E. (2006). La edición de revistas cientificas: directrices, criterios y modelos de evaluación. Madrid: FECYT.

Navarro, F. A. (2001). El inglés, idioma internacional de la medicina: causas y consecuencias de un fenómeno actual. Panace, 2(3), 35-51.

Oliver, F. C. (2009). Disseminação do conhecimento e periódicos científicos: contribuiçôes para o debate em terapia ocupacional. Cadernos de Terapia Ocupacional da UFSCar, 17(Suppl. Esp.), 69-85.

Organización de las Naciones Unidas - ONU. (2021). Estados membros. Recuperado el 10 de enero de 2020, de https://www.un.org/es/about-us/member-states.

Redalyc. (2020). Catálogo de revistas de Revistas Científicas de América Latina, el Caribe, España y Portugal. Recuperado el 10 de enero de 2020, de https://www.redalyc.org/

Rodger, S., McKenna, K., \& Brown, T. (2007). Quality and impact of occupational therapy journals: authors' perspectives. Australian Occupational Therapy Journal, 54(3), 174-184. http://dx.doi.org/10.1111/j.1440-1630.2007.00595.x.

Sobrido, M., Talavera, M. A., \& Gutiérrez, U. (2017). El artículo cientifico en terapia ocupacional. Madrid: Síntesis.

Talavera-Valverde, M. A. (2007). El legado de Barton. TOG, 2(1), 161-209.

Vasen, F., \& Vilchis, I. J. (2017). Sistemas nacionales de clasificación de revistas científicas en América Latina: tendencias recientes e implicaciones para la evaluación académica en ciencias sociales. Revista Mexicana de Ciencias Politicas y Sociales, 62(231), 199-228. http://dx.doi.org/10.1016/S0185-1918(17)30043-0.

World Federation of Occupational Therapists - WFOT. (2016). Minimum standards for the education of occupational therapists revised 2016. United Kingdom: WFOT.

\section{Contribución de los Autores}

Conceptualización: María Sobrido-Prieto. Recolección de datos, análisis formal, investigación, administración de proyectos, software, supervisión, validación, visualización, redacción de borradores originales y redacción, revisión y edición: María Sobrido-Prieto, Ana-Isabel Souto-Gómez y Miguel-Ángel TalaveraValverde. Todos los autores han leído y aceptado la version final del texto.

\section{Autor para la correspondencia}

Miguel-Ángel Talavera-Valverde e-mail: miguel.angel.talavera.valverde@udc.es

\section{Editor de sección}

Prof. Dr. Rodolfo Morrison 
Artículo Original

\title{
Un estudio descriptivo de la presencia, visibilidad y calidad de las revistas de terapia ocupacional
}

\author{
Um estudo descritivo da presença, visibilidade e qualidade dos \\ periódicos de terapia ocupacional
}

\section{A descriptive study of the presence, visibility and quality of occupational therapy journals}

\author{
María Sobrido-Prieto $^{a}$ (D), Miguel-Ángel Talavera-Valverde ${ }^{a}$ (D), Ana-Isabel Souto-Gómez ${ }^{b}$ (D) \\ aUniversidade da Coruña, A Coruña, Galicia, Espanha. \\ bUniversidade de Santiago de Compostela, Santiago de Compostela, Espanha.
}

Cómo citar: Sobrido-Prieto, M., Talavera-Valverde, M. A., \& Souto-Gómez, A. I. (2021). Un estudio descriptivo de la presencia, visibilidad y calidad de las revistas de terapia ocupacional. Cadernos Brasileiros de Terapia Ocupacional, 29, e2918. https://doi.org/10.1590/2526-8910.ctoAO2205

\begin{abstract}
$\underline{\text { Resumen }}$
Con el presente artículo intentamos conocer las revistas actuales de terapia ocupacional, así como la visibilidad y calidad de las mismas. La selección de la muestra se realizó a partir de la base de datos ULRICH y los catálogos Latindex y C17. La búsqueda se completó con un rastreo en la red. Los criterios de inclusión fueron revistas científicas revisadas por pares y activas en el momento de la consulta. Del listado final se analizaron las siguientes variables: visibilidad (se tuvo en cuenta el idioma de publicación y presencia en bases de datos internacionales -ERIC, Psychinfo, Medline, Scopus, OTDBASE, CINAHL and Web of Science), accesibilidad (suscripción, gratuidad y Open Access,) e indicadores de calidad (editorial responsable y factor de impacto). 43 revistas cumplieron los criterios. Respecto a la visiblidad el 69,8\% estaban indexadas en al menos una base de datos, siendo la de mayor representatividad, la base de datos propia de la disciplina, seguidas de las de ciencias de la salud, multidisciplinares y en menor medida las de ciencias sociales. El 25,6\% están incorporadas al movimiento Open Access y el 18,6\% son Free Access. Respecto al prestigio, un 20,9\% incorporadas a JCR y un $41,9 \%$ a SJR. Tras analizar cinco criterios de calidad, únicamente Hong Kong Journal of Occupational Therapy, Irish Journal of Occupational Therapy $y$ Occupational Therapy International los cumplen. Este estudio muestra la realidad de las revistas específicas de la especialidad, así como sus fortalezas y debilidades.
\end{abstract}


Errata

\title{
ERRATA: Un estudio descriptivo de la presencia, visibilidad y calidad de las revistas de terapia ocupacional
}

\author{
María Sobrido-Prieto ${ }^{\mathrm{a}}$ (D), Miguel-Ángel Talavera-Valverde ${ }^{\mathrm{a}}$ (D), Ana-Isabel Souto-Gómez ${ }^{\mathrm{b}}$ (D) \\ ${ }^{a}$ Universidade da Coruña, A Coruña, Galicia, Espanha.

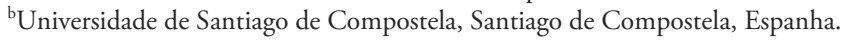

En el artículo "Un estudio descriptivo de la presencia, visibilidad y calidad de las revistas de terapia ocupacional”, DOI número https://doi.org/10.1590/25268910.ctoAO2205, publicado en Cadernos Brasileiros de Terapia Ocupacional, vol. 29, e2918, 2021,

En las páginas 9 y 10, donde se lee:

Tabla 2. Solapamiento de revistas de terapia ocupacional en las bases de datos revisadas $(\mathrm{n}=43)$.

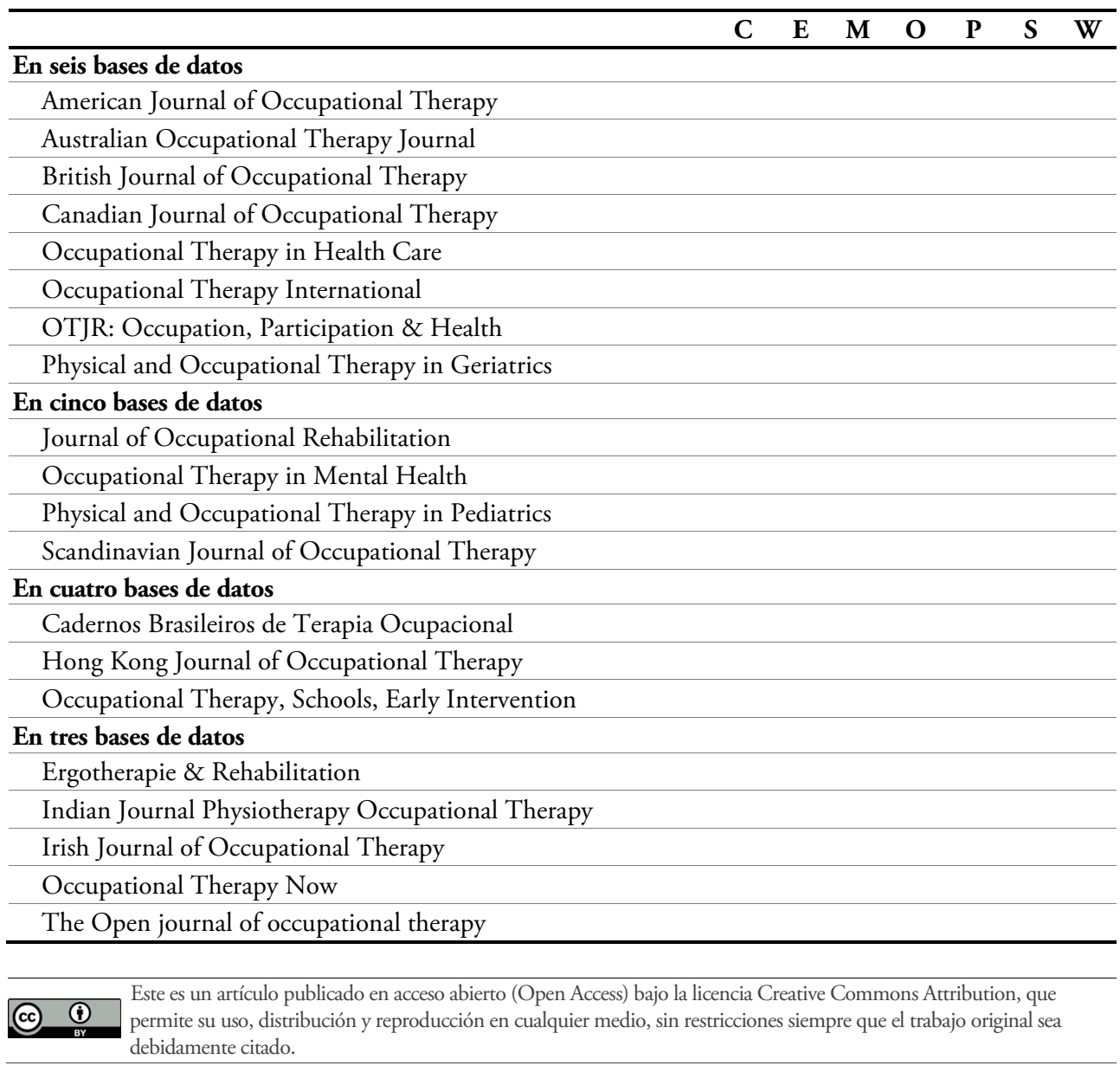


Tabla 2. Continuacion...

\section{En dos bases de datos}

Ergoscience

Indian Journal of Occupational Therapy

Revue Francophone de Recherche en Ergothérapie

New Zealand Journal of Occupational Therapy

South African Journal of Occupational Therapy

\section{En una base de datos}

Asian Journal of Occupational Therapy

ErgOThérapies

Israeli Journal of Occupational Therapy

TOG. Terapia Ocupacional Galicia*

World Federation of Occupational Therapists Bulletin

\section{En ninguna base de datos}

Annals of International Occupational Therapy

TJPRC:IJPOT

Journal of Korean Society of Occupational Therapy

Journal of Occupational Therapy Education

Physiotherapy and Occupational Therapy Journal

Revista Argentina de Terapia Ocupacional

Revista Asociación Española de Terapia Ocupacional

Revista Chilena de Terapia Ocupacional

Revista de Estudiantes de Terapia Ocupacional

Revista de Terapia Ocupacional de la USP

Revista Interinstitucional Brasileira Terapia Ocupacional

Revista Ocupación Humana

Japanese Occupational Therapy Research

C: CINAHL; E: ERIC; M: Medline; O: OTDBASE; P: Psycinfo; S: Scopus; W: WOS: Web of Science; TOG.

Terapia Ocupacional Galicia*: TOG (A Coruña).

\section{Debe leerse:}

Tabla 2. Solapamiento de revistas de terapia ocupacional en las bases de datos revisadas $(\mathrm{n}=43)$.

\begin{tabular}{|c|c|c|c|c|c|c|c|}
\hline & C & $\mathbf{E}$ & $\mathbf{M}$ & $\mathbf{O}$ & $\mathbf{P}$ & $\mathbf{S}$ & $\mathbf{W}$ \\
\hline \multicolumn{8}{|l|}{ En seis bases de datos } \\
\hline American Journal of Occupational Therapy & $\mathrm{X}$ & & $\mathrm{X}$ & $\mathrm{X}$ & $\mathrm{X}$ & $\mathrm{X}$ & $\mathrm{X}$ \\
\hline Australian Occupational Therapy Journal & $\mathrm{X}$ & & $\mathrm{X}$ & $\mathrm{X}$ & $\mathrm{X}$ & $\mathrm{X}$ & $\mathrm{X}$ \\
\hline British Journal of Occupational Therapy & $\mathrm{X}$ & & $\mathrm{X}$ & $\mathrm{X}$ & $\mathrm{X}$ & $\mathrm{X}$ & $\mathrm{X}$ \\
\hline Canadian Journal of Occupational Therapy & $\mathrm{X}$ & & $\mathrm{X}$ & $\mathrm{X}$ & $\mathrm{X}$ & $\mathrm{X}$ & $\mathrm{X}$ \\
\hline Occupational Therapy in Health Care & $\mathrm{X}$ & & $\mathrm{X}$ & $\mathrm{X}$ & $\mathrm{X}$ & $\mathrm{X}$ & $\mathrm{X}$ \\
\hline Occupational Therapy International & $\mathrm{X}$ & & $\mathrm{X}$ & $\mathrm{X}$ & $\mathrm{X}$ & $\mathrm{X}$ & $\mathrm{X}$ \\
\hline OTJR: Occupation, Participation \& Health & $\mathrm{X}$ & & $\mathrm{X}$ & $\mathrm{X}$ & $\mathrm{X}$ & $\mathrm{X}$ & $\mathrm{X}$ \\
\hline Physical and Occupational Therapy in Geriatrics & $\mathrm{X}$ & & $\mathrm{X}$ & $\mathrm{X}$ & $\mathrm{X}$ & $\mathrm{X}$ & $\mathrm{X}$ \\
\hline \multicolumn{8}{|l|}{ En cinco bases de datos } \\
\hline Journal of Occupational Rehabilitation & $\mathrm{X}$ & & $\mathrm{X}$ & & $\mathrm{X}$ & $\mathrm{X}$ & $\mathrm{X}$ \\
\hline Occupational Therapy in Mental Health & $\mathrm{X}$ & & $\mathrm{X}$ & $\mathrm{X}$ & & $\mathrm{X}$ & $\mathrm{X}$ \\
\hline Physical and Occupational Therapy in Pediatrics & $\mathrm{X}$ & & $\mathrm{X}$ & $\mathrm{X}$ & & $\mathrm{X}$ & $\mathrm{X}$ \\
\hline
\end{tabular}


Tabla 2. Continuacion...

\begin{tabular}{|c|c|c|c|c|}
\hline Scandinavian Journal of Occupational Therapy & $\mathrm{X}$ & $\mathrm{X}$ & $\mathrm{X}$ & $\mathrm{X} \quad \mathrm{X}$ \\
\hline \multicolumn{5}{|l|}{ En cuatro bases de datos } \\
\hline Cadernos Brasileiros de Terapia Ocupacional & $\mathrm{X}$ & & $\mathrm{X}$ & $\mathrm{X} X$ \\
\hline Hong Kong Journal of Occupational Therapy & $\mathrm{X}$ & $\mathrm{X}$ & & $\mathrm{X} X$ \\
\hline Occupational Therapy, Schools, Early Intervention & $\mathrm{X}$ & $\mathrm{X}$ & & $\mathrm{X} X$ \\
\hline \multicolumn{5}{|l|}{ En tres bases de datos } \\
\hline Ergotherapie \& Rehabilitation & $\mathrm{X}$ & & $\mathrm{X}$ & $\mathrm{X}$ \\
\hline Indian Journal Physiotherapy Occupational Therapy & $\mathrm{X}$ & $\mathrm{X}$ & $\mathrm{X}$ & \\
\hline Irish Journal of Occupational Therapy & $\mathrm{X}$ & & $\mathrm{X}$ & $\mathrm{X}$ \\
\hline Occupational Therapy Now & $\mathrm{X}$ & & $\mathrm{X}$ & $\mathrm{X}$ \\
\hline The Open journal of occupational therapy & $\mathrm{X}$ & $\mathrm{X}$ & $\mathrm{X}$ & \\
\hline \multicolumn{5}{|l|}{ En dos bases de datos } \\
\hline Ergoscience & $\mathrm{X}$ & & $\mathrm{X}$ & \\
\hline Indian Journal of Occupational Therapy & $\mathrm{X}$ & & $\mathrm{X}$ & \\
\hline Revue Francophone de Recherche en Ergothérapie & $\mathrm{X}$ & & $\mathrm{X}$ & \\
\hline New Zealand Journal of Occupational Therapy & $\mathrm{X}$ & & $\mathrm{X}$ & \\
\hline South African Journal of Occupational Therapy & $\mathrm{X}$ & & $\mathrm{X}$ & \\
\hline \multicolumn{5}{|l|}{ En una base de datos } \\
\hline Asian Journal of Occupational Therapy & & $\mathrm{X}$ & & \\
\hline ErgOThérapies & & & $\mathrm{X}$ & \\
\hline Israeli Journal of Occupational Therapy & & & $\mathrm{X}$ & \\
\hline TOG. Terapia Ocupacional Galicia* & & & $\mathrm{X}$ & \\
\hline World Federation of Occupational Therapists Bulletin & & & $\mathrm{X}$ & \\
\hline \multicolumn{5}{|l|}{ En ninguna base de datos } \\
\hline \multicolumn{5}{|l|}{ Annals of International Occupational Therapy } \\
\hline \multicolumn{5}{|l|}{ TJPRC:IJPOT } \\
\hline \multicolumn{5}{|l|}{ Journal of Korean Society of Occupational Therapy } \\
\hline \multicolumn{5}{|l|}{ Journal of Occupational Therapy Education } \\
\hline \multicolumn{5}{|l|}{ Physiotherapy and Occupational Therapy Journal } \\
\hline \multicolumn{5}{|l|}{ Revista Argentina de Terapia Ocupacional } \\
\hline \multicolumn{5}{|l|}{ Revista Asociación Española de Terapia Ocupacional } \\
\hline \multicolumn{5}{|l|}{ Revista Chilena de Terapia Ocupacional } \\
\hline \multicolumn{5}{|l|}{ Revista de Estudiantes de Terapia Ocupacional } \\
\hline \multicolumn{5}{|l|}{ Revista de Terapia Ocupacional de la USP } \\
\hline \multicolumn{5}{|l|}{ Revista Interinstitucional Brasileira Terapia Ocupacional } \\
\hline \multicolumn{5}{|l|}{ Revista Ocupación Humana } \\
\hline Japanese Occupational Therapy Research & & & & \\
\hline
\end{tabular}

C: CINAHL; E: ERIC; M: Medline; O: OTDBASE; P: Psycinfo; S: Scopus; W: WOS: Web of Science; TOG. Terapia Ocupacional Galicia*: TOG (A Coruña). 
En las páginas 13 y 14, donde se lee:

Tabla 4. Tabla resumen de las variables analizadas.

ID $\quad$ BD $\quad$ OA $\quad$ F:I

American Journal of Occupational Therapy

Annals of International Occupational Therapy

Asian Journal of Occupational Therapy

Australian Occupational Therapy Journal

British Journal of Occupational Therapy

Cadernos Brasileiros de Terapia Ocupacional*

Canadian Journal of Occupational Therapy**

Ergoscience

Ergotherapie und Rehabilitation

ErgOThérapies

Hong Kong Journal of Occupational Therapy

Indian Journal of Occupational Therapy

Indian Journal of Physiotherapy and Occupational Therapy

International Journal of Physiotherapy \& Occupational Therapy

Irish Journal of Occupational Therapy

Israeli Journal of Occupational Therapy

Japanese Occupational Therapy Research

Journal of Korean Society of Occupational Therapy

Journal of Occupational Rehabilitation

Journal of Occupational Therapy Education

Journal of Occupational Therapy, Schools, and Early Intervention

New Zealand Journal of Occupational Therapy

Occupational Therapy in Health Care

Occupational Therapy in Mental Health

Occupational Therapy International

Occupational Therapy Now**

OTJR: Occupation, Participation \& Health

Physical and Occupational Therapy in Geriatrics

Physical and Occupational Therapy in Pediatrics

Physiotherapy and Occupational Therapy Journal

Revista Argentina de Terapia Ocupacional

Revista Asociación Española de Terapia Ocupacional

Revista Chilena de Terapia Ocupacional

Revista de Estudiantes de Terapia Ocupacional

Revista de Terapia Ocupacional de la USP

Revista Interinstitucional Brasileira Terapia Ocupacional

Revista Ocupación Humana

Revue Francophone de Recherche en Ergothérapie

Scandinavian Journal of Occupational Therapy

South African Journal of Occupational Therapy

The Open journal of occupational therapy

TOG. Terapia Ocupacional Galicia*

World Federation of Occupational Therapists Bulletin

ID: idioma; Ing: inglés; BD: revista incluida en Bases de datos; OA: revista incluida en Open Access; F:I: revista con factor de impacto JCR o SJR; *Revista que se publica en Portugués/Castellano/Inglés; ${ }^{* *}$ Revista que se publica en Inglés/Francés; Revista de Terapia Ocupacional de la USP: Revista de Terapia Ocupacional da Universidade de São Paulo. *Continuación de la versión impresa. TOG. Terapia Ocupacional Galicia*: TOG (A Coruña). 


\section{Debe leerse:}

Tabla 4. Tabla resumen de las variables analizadas.

\begin{tabular}{|c|c|c|c|c|}
\hline & $\begin{array}{l}\text { ID } \\
\text { Ing }\end{array}$ & BD & OA & F:I \\
\hline American Journal of Occupational Therapy & $\mathrm{X}$ & $\mathrm{X}$ & & $\mathrm{X}$ \\
\hline Annals of International Occupational Therapy & $\mathrm{X}$ & & $\mathrm{X}$ & \\
\hline Asian Journal of Occupational Therapy & $\mathrm{X}$ & $\mathrm{X}$ & $\mathrm{X}$ & \\
\hline Australian Occupational Therapy Journal & $\mathrm{X}$ & $\mathrm{X}$ & & $\mathrm{X}$ \\
\hline British Journal of Occupational Therapy & $\mathrm{X}$ & $\mathrm{X}$ & & $\mathrm{X}$ \\
\hline Cadernos Brasileiros de Terapia Ocupacional* & & $\mathrm{X}$ & $\mathrm{X}$ & \\
\hline Canadian Journal of Occupational Therapy** & & $\mathrm{X}$ & & $\mathrm{X}$ \\
\hline Ergoscience & & $\mathrm{X}$ & & \\
\hline Ergotherapie und Rehabilitation & & $\mathrm{X}$ & & $\mathrm{X}$ \\
\hline ErgOThérapies & & $\mathrm{X}$ & & \\
\hline Hong Kong Journal of Occupational Therapy & $\mathrm{X}$ & $\mathrm{X}$ & $\mathrm{X}$ & $\mathrm{X}$ \\
\hline Indian Journal of Occupational Therapy & $\mathrm{X}$ & $\mathrm{X}$ & & \\
\hline Indian Journal of Physiotherapy and Occupational Therapy & $\mathrm{X}$ & $\mathrm{X}$ & & $\mathrm{X}$ \\
\hline International Journal of Physiotherapy \& Occupational Therapy & $\mathrm{X}$ & & & \\
\hline Irish Journal of Occupational Therapy & $\mathrm{X}$ & $\mathrm{X}$ & $\mathrm{X}$ & $\mathrm{X}$ \\
\hline Israeli Journal of Occupational Therapy & & $\mathrm{X}$ & & \\
\hline \multicolumn{5}{|l|}{ Japanese Occupational Therapy Research } \\
\hline \multicolumn{5}{|l|}{ Journal of Korean Society of Occupational Therapy } \\
\hline Journal of Occupational Rehabilitation & $\mathrm{X}$ & $\mathrm{X}$ & & $\mathrm{X}$ \\
\hline Journal of Occupational Therapy Education & $\mathrm{X}$ & & $\mathrm{X}$ & \\
\hline Journal of Occupational Therapy, Schools, and Early Intervention & $\mathrm{X}$ & $\mathrm{X}$ & & $\mathrm{X}$ \\
\hline New Zealand Journal of Occupational Therapy & $\mathrm{X}$ & $\mathrm{X}$ & & \\
\hline Occupational Therapy in Health Care & $\mathrm{X}$ & $\mathrm{X}$ & & $\mathrm{X}$ \\
\hline Occupational Therapy in Mental Health & $\mathrm{X}$ & $\mathrm{X}$ & & $\mathrm{X}$ \\
\hline Occupational Therapy International & $\mathrm{X}$ & $\mathrm{X}$ & $\mathrm{X}$ & $\mathrm{X}$ \\
\hline Occupational Therapy Now** & & $\mathrm{X}$ & & $\mathrm{X}$ \\
\hline OTJR: Occupation, Participation \& Health & $\mathrm{X}$ & $\mathrm{X}$ & & $\mathrm{X}$ \\
\hline Physical and Occupational Therapy in Geriatrics & $\mathrm{X}$ & $\mathrm{X}$ & & $\mathrm{X}$ \\
\hline Physical and Occupational Therapy in Pediatrics & $\mathrm{X}$ & $\mathrm{X}$ & & $\mathrm{X}$ \\
\hline Physiotherapy and Occupational Therapy Journal & $\mathrm{X}$ & & & \\
\hline \multicolumn{5}{|l|}{ Revista Argentina de Terapia Ocupacional } \\
\hline \multicolumn{5}{|l|}{ Revista Asociación Española de Terapia Ocupacional } \\
\hline Revista Chilena de Terapia Ocupacional & & & $\mathrm{X}$ & \\
\hline \multicolumn{5}{|l|}{ Revista de Estudiantes de Terapia Ocupacional } \\
\hline \multicolumn{5}{|l|}{ Revista de Terapia Ocupacional de la USP } \\
\hline Revista Interinstitucional Brasileira Terapia Ocupacional & & & $\mathrm{X}$ & \\
\hline Revista Ocupación Humana & & & $\mathrm{X}$ & \\
\hline Revue Francophone de Recherche en Ergothérapie & & $\mathrm{X}$ & $\mathrm{X}$ & \\
\hline Scandinavian Journal of Occupational Therapy & $\mathrm{X}$ & $\mathrm{X}$ & & $\mathrm{X}$ \\
\hline South African Journal of Occupational Therapy & $\mathrm{X}$ & $\mathrm{X}$ & & \\
\hline The Open journal of occupational therapy & $\mathrm{X}$ & $\mathrm{X}$ & $\mathrm{X}$ & \\
\hline TOG. Terapia Ocupacional Galicia* & & $\mathrm{X}$ & $\mathrm{X}$ & \\
\hline \multirow[t]{2}{*}{ World Federation of Occupational Therapists Bulletin } & $\mathrm{X}$ & $\mathrm{X}$ & & \\
\hline & $\mathrm{X}$ & $\mathrm{X}$ & & \\
\hline
\end{tabular}

ID: idioma; Ing: inglés; BD: revista incluida en Bases de datos; OA: revista incluida en Open Access; F:I: revista con factor de impacto JCR o SJR; *Revista que se publica en Portugués/Castellano/Inglés; ${ }^{* *}$ Revista que se publica en Inglés/Francés; Revista de Terapia Ocupacional de la USP: Revista de Terapia Ocupacional da Universidade de São Paulo. *Continuación de la versión impresa. TOG. Terapia Ocupacional Galicia*: TOG (A Coruña). 